\title{
DNA nanorobots seek and destroy
}

Due to the potential of DNA molecules as substrates for the production of molecular machines with biological functionality, there is much interest in using DNA-based nanorobotic systems for the targeted delivery of therapeutic payloads to tumours. A new study reports the development of autonomous 'DNA nanorobots' that transport and deliver protease payloads to tumour-associated blood vessels, eliciting a cascade of coagulation, vascular occlusion and necrosis.

To facilitate the delivery of the coagulation protease thrombin to tumours, DNA origami was used to develop a thrombin-conjugated DNA nanotube that shields the protease from circulating substrates. DNA nanotubes were also hybridized to predesigned DNA aptamers that recognize nucleolin, which is selectively expressed on tumourassociated vascular endothelial cells. Upon nucleolin binding, these thrombinloaded nanorobots (Th-nanorobots) reconfigured into sheets, exposing the thrombin payload.

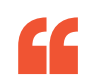

The findings demonstrate the early therapeutic potential of DNA nanorobotic systems 3
Indeed, mouse plasma rapidly coagulated in vitro when mixed with Th-nanorobots and human umbilical vein endothelial cells.

The investigators first evaluated the ability of Th-nanorobots to target tumour vessels in vivo. Following intravenous injection, Th-nanorobots progressively accumulated within orthotopic MDA-MB-231 breast cancer tumours in mice. Moreover, Th-nanorobots colocalized with the endothelial cell marker CD34, sugges-

ting their selective association with the vascular epithelium. Importantly, Th-nanorobot treatment induced the aggregation of activated platelets and progressive thrombosis in orthotopic tumours, whereas thrombosis was limited or absent in tumour-bearing mice treated with various controls. Next, the therapeutic effect of Th-nanorobots on tumour growth in vivo was assessed in several mouse models. Advanced tumour necrosis was detected in MDA-MB-231 tumours from Th-nanorobottreated mice but not in the control groups. Moreover, the growth of established MDA-MB-231 tumours was markedly lower in mice treated with Th-nanorobots than with controls. Interestingly, therapeutic efficacy was greater in the B16-F10 melanoma mouse model, in which Th-nanorobot treatment induced complete regression of established tumours in three out of eight mice and abrogated liver metastasis. In mice bearing established SK-OV3 ovarian cancer xenografts (which are poorly vascularized), Th-nanorobot treatment also prolonged survival, illustrating that limited tumour permeability does not impede therapeutic efficacy. Additionally, in a doxycycline-inducible $\mathrm{Kras}^{\mathrm{G} 12 \mathrm{D}}$ lung cancer mouse model, which recapitulates the course of human lung cancer, magnetic resonance imaging revealed that Th-nanorobots markedly reduced tumour growth compared with controls. Th-nanorobots were also shown to be safe and immunologically inert in mice and Bama miniature pigs.

The findings demonstrate the early therapeutic potential of DNA nanorobotic systems as vehicles for the delivery of biochemical moieties that are not amenable as conventional therapeutics owing to their extremely high or low bioactivities, in order to achieve targeted tumour therapy.

Conor A. Bradley

This article is modified from the original in Nat. Rev. Cancer (http://dx.doi.org/10.1038/nrc.2018.19)

ORIGINAL ARTICLE Li, S. et al. A DNA nanorobot functions as a cancer therapeutic in response to a molecular trigger in vivo. Nat. Biotechnol. https://doi.org/10.1038/nbt.4071 (2018) 